\title{
Abwarten ist keine Lösung
}

VON MATTHIAS KÖNIG,

HARTMUT CLAUSEN UND

CHRISTOPH SCHANK

Dr. Matthias König und Hartmut

Clausen arbeiten als Unterneh-

mensberater bei der Hamburger

Beratungsfirma akquinet business

consulting $\mathrm{GmbH}$, die auf die

Sozialbranche spezialisiert ist.

Zuvor hatten beide Führungspo-

sitionen in der Sozialwirtschaft

inne. Dr. Christoph Schank ist Be-

rater am Institut für werteorien-

tierte Unternehmensführung und

wissenschaftlicher Mitarbeiter an der Universität St.Gallen.

www.akquinet.de

www.iwe.unisg.ch

\author{
Die Sozialwirtschaft ist massiv von einem \\ Fachkräftemagel betroffen. Die Unternehmen und \\ Organisationen schreiben diesem Problemen zwar \\ einen hohen Stellenwert zu, begegnen ihm aber \\ nicht durch konsequentes Handeln, meint eine \\ empirische Studie bei Betrieben der Sozialwirtschaft.
}

Der Fachkräftemangel in der Sozialwirtschaft ist fast schon ein alter Hut. Die Nachfrage nach sozialen Dienstleistungen wird künftig steigen, in den Pflegeberufen wegen der Alterung der Gesellschaft, in der Behindertenhilfe aufgrund des medizinischen Fortschritts oder in den Kindertagesstätten weil gesetzliche Garantien gegeben wurden. Und gleichzeitig sinkt, wegen des demografischen Wandels, die Anzahl der jungen Menschen, die für den Arbeitsmarkt und dem dualen Ausbildungssystem zur Verfügung stehen.

Die Diagnose beruht auf Prognosen, die auch angezweifelt werden. So werde eine Erhöhung der Produktivität vernachlässigt oder die stärkere Zuwanderung von ausländischen Fachkräften unterschätzt oder Fachkräfte, die bisher nicht dem Arbeitsmarkt zur Verfügung stehen (z. B. weil sie ihre Kinder erziehen), nicht genügend aktiviert.

Sind die Unternehmen und Organisationen der Sozialwirtschaft auf den Fachkräftemangel und seine Abwehr vorbereitet? In der öffentlichen Diskussion steht der akute Fachkräftemangel in den Pflegeberufen im Mittelpunkt. Wie sieht es in den anderen Handlungsfeldern der Sozialwirtschaft aus? Sind auch sie schon betroffen oder erst in Zukunft?

Diese und ähnliche Fragen wollten wir mit einer empirischen Studie zur aktuellen Situation des Fachkräftemangels in der Sozialwirtschaft beantworten. (1) Die Untersuchung möchte einen Beitrag zur Versachlichung der Diskussion leisten und Impulse für das Handeln der Unternehmen und Organisationen geben. An der Befragung im Februar 2012 haben 133 Unternehmen und Organisationen der Sozialwirtschaft teilgenommen. Sie bilden einen repräsentativen Querschnitt durch die Sozialwirtschaft. Von der Unternehmensgröße, der Rechtsform oder den inhaltlichen Handlungsfeldern sind alle Ausprägungen vorhanden, auch in der für die Sozialwirtschaft typischen Besonderheit.

\section{Fachkräftemangel ist weit verbreitet}

Der Fachkräftemangel durchzieht die gesamte Sozialwirtschaft. Im Durchschnitt haben $82 \%$ der befragten Unternehmen und Organisationen in den letzten zwei Jahren Probleme bei der Besetzung offener Stellen gehabt.

Die ambulante Pflege liegt mit $94 \%$ an der Spitze, gefolgt von den Beratungsstellen (92\%) und der Kinder-, Jugend-, Familienhilfe mit $89 \%$. Andere große Bereiche der Sozialwirtschaft wie die stationäre Altenpflege $(88 \%)$ oder die Behindertenhilfe (84\%) liegen ebenfalls über dem Durchschnitt. Dieses Ergebnis ist auch unabhängig von der Größe der Unternehmen und Organisationen oder ihrer Rechtsformen.

Untermauert wird die allgemeine Einschätzung von der Anzahl der aktuell offenen Stellen. Länger als drei Mona- 
te ist mindestens eine Stelle bei $81 \%$ der Unternehmen und Organisationen mit Fachkräftemangel nicht besetzt. Mehr als elf Stellen bleiben bei $12 \%$ der Befragten offen. Der Grund für die Nichtbesetzung von Stellen ist mit weitem Abstand die unzureichende Anzahl von Bewerbenden (68 \%). In der Sozialwirtschaft liegt also wirklich ein Mangel vor.

Kommt es dann zu einer Bewerberauswahl, liegt die häufigste Schwierigkeit in den unzureichenden fachlichen Kenntnissen (63\%) der Bewerberinnen und Bewerber (2), gefolgt von den nicht passenden persönlichen Kompetenzen (56\%). Die meisten Probleme bei der Besetzung offener Stellen gibt es aktuell bei der Suche nach ausgebildeten Fachkräften $(58 \%)$ und Führungskräften $(36 \%)$. Für Hilfsstellen oder nicht-pflegerische Fachkräfte können die Stellen mit weniger Problemen besetzt werden. Der vorliegende Mangel ist demnach einer der Fachkräfte.

\section{Verantwortung wird bei Politik und Tarifparteien gesehen}

Auch wenn die Prognosen zum Fachkräftemangel für die Zukunft vielleicht hinterfragt werden können, so ist er für die Breite der Sozialwirtschaft bereits Realität. Wie geht sie damit um?

Zunächst sehen die Unternehmen und Organisationen sich selbst nicht als hauptsächlichen Akteur gegen den Fachkräftemangel. Diese Rolle wird der Politik zugeteilt. Ihr wird mit $87 \%$ eine große Verantwortung zugeschrieben, ge- folgt von den Tarifparteien (75\%) und den Unternehmen und Organisationen selbst (69\%). Die Wohlfahrtsverbände werden nur von $60 \%$ in der Verantwortung gesehen.

Die Hauptrolle der Politik steht im Zusammenhang mit der stärksten Forderung an sie, für eine bessere Refinanzierung für höhere Löhne (86\%) zu sorgen. Daraus dürfte sich auch die hohe Wertung für die Tarifparteien erklären. Der Rahmen für die Arbeitsbedingungen in der Sozialwirtschaft wird von diesen beiden Akteuren gesetzt. Sie haben einen erheblichen Einfluss auf den Fachkräftemangel. Und hier scheint ein erheblicher Handlungsbedarf zu bestehen.

Obwohl die Unternehmen und Organisationen der Sozialwirtschaft sich eher eine Nebenrolle zubilligen, ist der Fachkräftemangel doch bei ihnen angekommen. Für $75 \%$ hat er einen hohen Stellenwert in dem operativen Handeln und bei nur unmerklich geringeren $72 \%$ ist der Stellenwert für das strategische Handeln hoch. Die Bedeutung des Fachkräftemangels für die Unternehmen und Organisationen ist von der aktuellen Situation getrieben. Suchen sie Fachkräfte und sind viele Stellen offen, ist auch der Stellenwert des Fachkräftemangels für das Handeln hoch.

Deutlich wird das auch bei Unternehmen und Organisationen mit einem hohen operativen Stellenwert für den Fachkräftemangel. Ihr Fokus bei den durchgeführten Maßnahmen ist auf diejenigen gerichtet, die direkt neue Fachkräfte zur Folge haben. Dies sind bei- spielsweise die Ansprache ausländischer Fachkräfte (Korrelation von 0,368) (3), die eigene Ausbildung von Fachkräften $(0,324)$ oder eine höhere Entlohnung (0,282), eine Maßnahme, die eher selten durchgeführt wird).

Die Unternehmen und Organisationen, die dem Fachkräftemangel einen strategischen Stellenwert einräumen, setzen eher planende Instrumente um, die eine vorbereitende Wirkung haben. Beispiele hierfür sind eine intensivierte Karriereplanung $(0,282)$, die qualitative Personalplanung $(0,267)$ oder erst einmal das Aufstellen von Konzepten gegen den Fachkräftemangel $(0,225)$.

\section{Was Unternehmen und Organisationen tun wollen - und was nicht}

Der Fachkräftemangel hat für das Handeln der Unternehmen und Organisationen nach ihrer eigenen Einschätzung einen hohen Stellenwert. Wie konkretisiert sich das? Welche Maßnahmen werden umgesetzt und geplant, welchen Einfluss haben diese auf den Fachkräftemangel? Um hierauf Antworten geben zu können, haben wir diverse Maßnahmen aus den unterschiedlichen Bereichen eines Unternehmens abgefragt, die alle im Zusammenhang mit dem Fachkräftemangel stehen.

Grundsätzlich haben Unternehmen unterschiedliche Möglichkeiten, dem Fachkräftemangel zu begegnen. Die Maßnahmen lassen sich in Kategorien einteilen, wie dem Fachkräftemangel begegnet werden kann (vgl. Abb. 1). $\rightarrow$

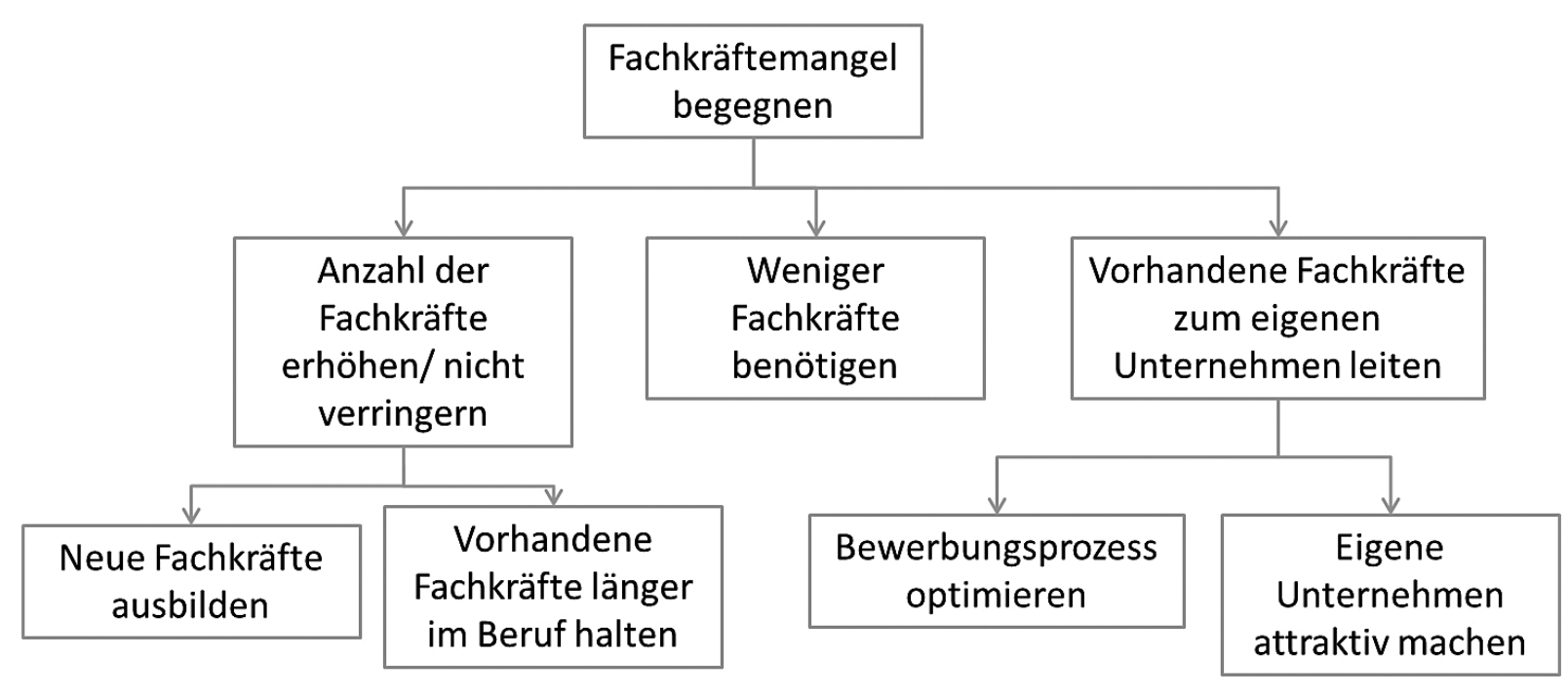

Abb. 1: Unternehmen und Organisationen haben grundsätzliche verschiedene Möglichkeiten, dem Fachkräftemangel zu begegnen. 


\begin{tabular}{|l|c|c|c|}
\hline Grundsätzliche Möglichkeit & Wird umgesetzt & $\begin{array}{c}\text { Soll (intensiver) } \\
\text { umgesetzt werden }\end{array}$ & $\begin{array}{c}\text { Zur Umsetzung nicht } \\
\text { vorgesehen }\end{array}$ \\
\hline Attraktiver für Bewerbende werden & $72 \%$ & $37 \%$ & $14 \%$ \\
\hline Bewerbungsprozess verbessern & $67 \%$ & $60 \%$ & $12 \%$ \\
\hline Fachkräfte länger im Beruf halten & $67 \%$ & $49 \%$ & $13 \%$ \\
\hline Weniger Fachkräfte benötigen & $59 \%$ & $31 \%$ & $29 \%$ \\
\hline Neue Fachkräfte & $54 \%$ & $36 \%$ & $30 \%$ \\
\hline
\end{tabular}

Abb. 2: Bekämpfung des Fachkräftemangels werden in der Sozialwirtschaft unterschiedlich stark genutzt (Mehrfachnennungen möglich).

Der direkteste Weg ist die Erhöhung der Anzahl der Fachkräfte. Dies kann beispielsweise geschehen durch die eigene Ausbildung von Fachkräften, die Weiterbildung für Berufsrückkehrer und für Quereinsteiger oder die Ansprache ausländischer Fachkräfte.

Der Pflegeberuf ist einer der Berufe in Deutschland mit der geringsten Verweildauer der Menschen in dem Beruf. Grund hierfür dürfte die hohe physische und psychische Belastung sein. Daraus ergibt sich der Ansatzpunkt, die in dem Beruf tätigen Fachkräfte länger im Beruf zu halten. Realisieren lässt sich das beispielsweise durch Maßnahmen des Gesundheitsschutzes, eine berufsbegleitende Qualifizierung oder eine alternsgerechte Personalentwicklung.

Der Fachkräftemangel kann auch verringert werden, wenn weniger Fachkräfte benötigt werden. So kann beispielsweise mit einer effizienteren Arbeitsorganisation oder durch Zielvereinbarungen die Produktivität erhöht werden.

Zwei weitere grundsätzliche Möglichkeiten stehen den Unternehmen und Organisationen zur Verfügung. Hierbei wird allerdings nicht das Angebot an Fachkräften erhöht oder die Nachfrage nach ihnen gesenkt, sondern sie sorgen dafür, dass die vorhandenen Fachkräfte zum eigenen Unternehmen kommen. Der eine Weg ist die Optimierung des Bewerbungsprozesses, einschließlich der Ansprache bei der Bewerbersuche; der andere Weg ist, das eigene Unternehmen für Bewerber attraktiver zu machen. Geschehen kann dies beispielsweise durch eine zeitgemäße Führung, durch (finanzielle) Zusatzleistungen, die Schaffung eines angenehmen Arbeitsklimas oder die Stärkung der Familienfreundlichkeit.

Wie nutzen die Unternehmen und Organisationen die grundsätzlichen Möglichkeiten? Die in der empirischen Studie erhobenen Maßnahmen haben wir den
Kategorien zugeordnet und Durchschnittswerte gebildet (vgl. Abb. 2).

Zunächst ist festzuhalten, dass von den Unternehmen und Organisationen der Sozialwirtschaft viele unterschiedliche Maßnahmen im großen Umfang umgesetzt werden. Erstaunlich sind aber die Prioritäten.

An erster Stelle stehen die Maßnahmen-Kategorien, die den allgemeinen Fachkräftemangel nicht beeinflussen, sondern auf das eigene Unternehmen und Organisation bezogen sind. Ein optimierter Bewerbungsprozess schafft aber keine neuen Fachkräfte. Maßnahmen für neue Fachkräfte finden sich hingegen auf dem letzten Platz bei den durchgeführten Maßnahmen (54\%). Ausgeglichen werden soll dies nicht durch geplante Maßnahmen oder die Intensivierung bestehender. Nur 36\% der Unternehmen und Organisationen wollen zukünftig ihre Anstrengungen verstärken, die Anzahl der Fachkräfte zu erhöhen. Fast genauso viele (30\%)
Maßnahmen, die die Unternehmen und Organisationen der Sozialwirtschaft planen umzusetzen oder zu intensivieren, sind die Maßnahmen, die aus ihrer Sicht geeignet sind, dem Fachkräftemangel zu begegnen. In der Rangliste dieser »Goalgetter " finden sich keine Maßnahmen mit direkter Auswirkung auf den Fachkräftemangel. Zunächst geht es um die Personalbeschaffung mit einem zielgruppenspezifischen Marketing $(63 \%)$ und der Optimierung der Personalbeschaffung $(58 \%)$. Beides ist auf das einzelne Unternehmen und Organisation bezogen, ändert aber nichts am allgemeinen Fachkräftemangel. Konzepte und Strategien zum Fachkräftemangel zu erstellen haben sich $58 \%$ der Unternehmen und Organisationen der Sozialwirtschaft vorgenommen. Sie wollen sich also erst noch grundsätzlich mit dem Thema auseinandersetzen. Hier wird ein klarer Handlungsbedarf deutlich. Und auch verständlich, warum die Unternehmen und Organisationen

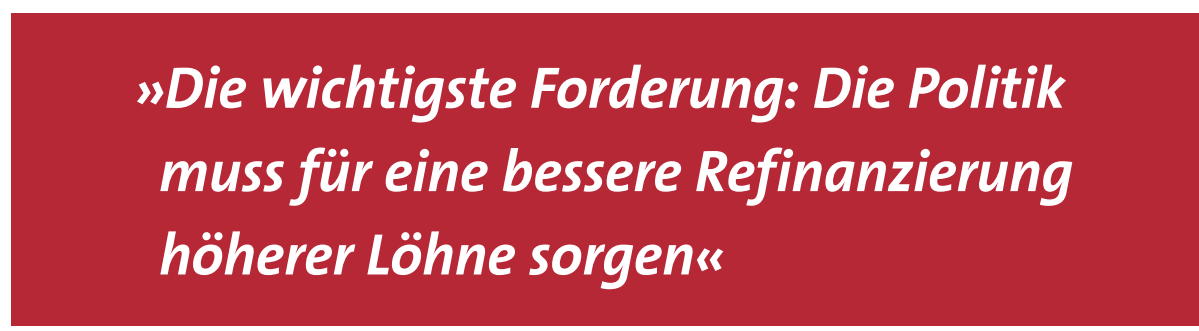

wollen sich überhaupt nicht mit dem Thema beschäftigen. Bei dem Maßnahmenbündel, durch erhöhte Produktivität weniger Fachkräfte zu benötigen, sieht die Lage ähnlich aus. Dies erstaunt umso mehr, da dies auch Maßnahmen sind, die Kosten sparen.

Die Unternehmen und Organisationen der Sozialwirtschaft schreiben dem Fachkräftemangel für sich einen hohen Stellenwert zu, setzen es aber nicht in konsequentes Handeln um. Der Blick auf die Ranglisten der Einzel-Maßnahmen bestätigt dies. sich vornehmlich mit indirekten Maßnahmen gegen den Fachkräftemangel beschäftigen. Ein Erklärungsversuch ist der Handlungsdruck. Wie schon bei dem Stellenwert, so ist auch bei den Maßnahmen erkennbar, dass bei steigendem Handlungsdruck Maßnahmen mit einer direkten Wirkung umgesetzt werden.

Eine gegenteilige Rangliste führt Maßnahmen auf, die die Unternehmen und Organisationen der Sozialwirtschaft aktuell nicht umsetzen und auch nicht planen umzusetzen. Demnach 
müssten es die Maßnahmen sein, die ungeeignet sind, eine Wirkung in Bezug auf den Fachkräftemangel zu haben. Vielmehr sind es Maßnahmen, die für die Sozialwirtschaft untypisch sind - den Fachkräftemangel beeinflussen, könnten sie aber schon.

An der Spitze findet sich die verstärkte Nutzung von Zeitarbeit (61 \%) und die Ansprache ausländischer Fachkräfte $(58 \%)$. Die Zeitarbeit wird in der Sozialwirtschaft zu einem sehr geringen Teil eingesetzt. (4) Sie ist auch heftig umstritten. Punktuell genutzt, kann sie aber kurzfristig Engpässe überbrücken. Die Ansprache ausländischer Fachkräfte ist eine der Maßnahmen, auf die die Politik sich konzentriert. In der Sozialwirtschaft wird sie mehrheitlich abgelehnt. Beide Maßnahmen werden hauptsächlich in der stationären Pflege umgesetzt (Zeitarbeit: Korrelation von 0,453; ausländische Fachkräfte von 0,265). Die Weiterbildung für Berufsrückkehrer findet sich auch auf der Liste der nicht-gewollten Maßnahmen. Damit wird gerade in der Sozialwirtschaft ein großes Potential nicht genutzt. Die meisten der anderen Maßnahmen auf der »Giftliste« sind mit höheren finanziellen Aufwänden verbunden.

\section{Was nun?}

Der Fachkräftemangel ist zwar Realität und auch bei den Unternehmen und Organisationen der Sozialwirtschaft angekommen, aber der akute Handlungsdruck ist anscheinend nicht so hoch, dass eine der Notwendigkeit angemessene Beschäftigung mit dem Thema erfolgt.

Zudem sehen die Unternehmen und Organisationen hauptsächlich die Politik und die Tarifparteien in der Verantwortung. Deren Agieren ist aber mit höheren finanziellen Aufwendungen der öffentlichen Hand verbunden, worauf nicht vertraut werden sollte.

Der Fachkräftemangel kann nur dann abgeschwächt werden, wenn die Unternehmen und Organisationen selbst eine aktive Rolle übernehmen und verstärkt für neue Fachkräfte sorgen. Denn der Handlungsdruck wird steigen. Wird dann erst reagiert, wird dies zu Lasten der Dienstleistungsqualität und damit zu Lasten der Betreuten und Nutzer gehen.

\section{Anmerkungen}

(1) In diesem Artikel werden Ausschnitte der Ergebnisse dargestellt. Eine ausführliche Zusammenfassung der Studie ist unter fragebogen.bc@akquinet.de erhältlich.

(2) Der Fragebogen hat die übliche fünfpolige Likert-Skala verwendet. Im Folgenden sind in Prozent dargestellt jeweils die beiden höchsten Wertungen je Frage (aus der Skala 1-5 die Wertungen 4 und 5).

(3) Statistisch ausgedrückt haben wir den Zusammenhang über Korrelationen gemessen. Korrelationen können zwar keine Ursachen beweisen, aber Zusammenhänge zwischen (mindestens) zwei Antworten aufzeigen. Für dieses Beispiel: Die Teilnehmer, die den operativen Stellenwert hoch bewertet haben, haben auch die Maßnahme Ansprache ausländischer Fachkräfte hoch bewertet.

(4) Knapp 2,8 Millionen sozialversicherungspflichtigen Beschäftigten im Gesundheitsbereich stehen 16.350 in der Zeitarbeit gegenüber (Quelle: www.sueddeutsche.de vom 5.07.2012).

\section{Markenwert durch Mitarbeiter steigern}

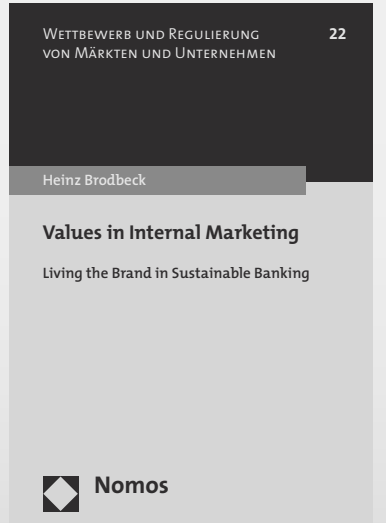

\author{
Values in Internal Marketing \\ Living the Brand in Sustainable Banking \\ Von Heinz Brodbeck \\ 2013, 269 S., brosch., 54,- €, ISBN 978-3-8487-0534-4 \\ (Wettbewerb und Regulierung von Märkten und Unternehmen, Bd. 22)
}

Der Autor legt eine neue Darstellung vor, wie wertebezogenes Mitarbeiterverhalten entsteht und wirkt. Er konzipiert ,Living the Brand' aus der Sicht nachhaltiger Wettbewerbsvorteile und erforscht das Modell im Bereich ökologischer Finanzservices. Erkenntnisse zur Steigerung des Markenwertes für Manager, Marketing-, HR-Fachleute, Studenten und Ökonomen.

Bestellen Sie jetzt telefonisch unter 07221/2104-37

Portofreie Buch-Bestellungen unter www.nomos-shop.de/21038

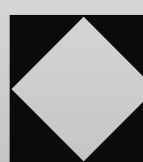

Nomos 